\title{
A Novel Operating-Point Driven Method for the Sizing of Analog IC
}

\author{
Bo Liu", Murat Pak ${ }^{2}$, Xuezhi Zheng ${ }^{1}$ and Georges Gielen ${ }^{1}$. \\ ${ }^{1}$ ESAT, K.U.Leuven, Leuven, Belgium and ${ }^{2}$ Bogazici University, Istanbul, Turkey \\ E-mail: ${ }^{1}\left\{\right.$ Bo.Liu, Xuezhi.Zheng, Georges.Gielen\}@esat.kuleuven.be, ${ }^{2}$ murat.pak@boun.edu.tr
}

\begin{abstract}
It is known that the operating-point driven (OPD) analog sizing methods have clear advantages compared with the sizing methods of directly using transistor width and length as the decision variables. However, new analog sizing algorithms using OPD technique in modern technologies have seldom been reported in recent years. One of the main reasons is that with the scaling down of the technologies, the transistor models are much more complex, which makes the available DC root solving algorithms and the look-up-table-based methods face significant challenges on accuracy, efficiency and memory requirements. Instead of solving the equations to find the width of transistors, interpolating in a pre-constructed look-up-table, or using regression methods, a novel method, called on-line interpolation operating-point driven (OIOPD), is proposed. OIOPD finds the width of the transistor by the interpolation of the width-current curve with already determined length and voltage biases. The lower and upper points to decide the interpolated value are generated by on-line simulations using the two extreme values of the width in a technology. Experimental results in $0.25 \mu \mathrm{m}, 0.18 \mu \mathrm{m}$ and $90 \mathrm{~nm}$ technologies show that OIOPD has 10 times improvement on accuracy, 3001100 times improvement on efficiency compared with the available methods. In addition, no extra memory (e.g. the memory to save the look-up table) is needed. These advantages make OIOPD suitable for operating-point driven analog sizing methods in modern technologies. A practical analog sizing example using OIOPD is also provided.
\end{abstract}

\section{INTRODUCTION}

Analog cell sizing methods can be classified into two main categories: width and length (W/L)-based methods [1,2] and operating-point driven (OPD)-based methods [3-5]. The former method uses the width and length as the design variables. The latter method, however, uses operating point as the design variables and the device dimensions (W) are determined out of it. OPD-based method is used much less than width and length-based method in recent literatures. However, it has been reported that the OPD-based methods have distinct advantages [3-5], which can be summarized as follows. Firstly, it highly relieves the convergence problem of electrical simulators, as a DC consistent solution cannot be found for some $\mathrm{W} / \mathrm{L}$ in the search space and these $\mathrm{W} / \mathrm{L}$ are difficult to be pruned beforehand [3]. Secondly, device operating constraints (e.g.

$V_{g s}>0$, transistor in the saturation region) [6] are self-contained in the generation of the candidate solutions in the OPD-based methods, which can ensure the devices to operate in the intended region. These constraints are obvious to the designer, but not to the optimization algorithm. For W/L-based methods, explicitly measurements and enforcements need to be added, which increase the number of constraints and make the optimization problem more difficult [5]. Thirdly, the design variable $\mathrm{W}$ often has a large range in W/L-based method, which adds high pressure to the search algorithm, especially when the circuit is complex. On the other hand, $\mathrm{W}$ is calculated from device biases (voltages, currents) in OPD-based methods, which decreases the search space, because voltage / current biases and L have small ranges. Fourthly, OPDbased methods allow the designer to reason in terms of voltages and currents and relieve him from the burden of determining device sizes [3].

OPD-based methods have such advantages, but why they seldom appear in recent literatures? An important reason is that with the scaling down of devices, the models are becoming more and more complex. According to our experiments (see Section IV), the available DC root solving algorithms and the look-up-tablebased methods face significant challenges on accuracy, efficiency and memory requirements.

To address these problems, a new OPD method, called on-line interpolation operating-point driven (OIOPD), is proposed. In OIOPD, the width of a transistor is computed by the interpolation of the width-current curve with a determined set of length and voltage biases. The two points $\left(W_{\min }, I_{\min }\right)$ and $\left(W_{\max }, I_{\max }\right)\left(W_{\min }\right.$ and $W_{\max }$ are the two extreme allowed values of $\mathrm{W}$ for a technology) are first simulated on-line, then the request $\mathrm{W}$ is computed by the SPLINE interpolation [10]. OIOPD has 10 times improvement on accuracy, 300-1100 times improvement on efficiency compared with the available methods. In addition, OIOPD need not to tune the parameters, e.g. the size of the LUT, the number of neurons in artificial neural network (ANN).

The remainder of the paper is organized as follows. Section II reviews the available OPD-based methods. Section III describes the proposed OIOPD method. Section IV compares OIOPD with the available methods in 3 technologies. Section V provides a practical example of complex analog circuit sizing using OIOPD in a $0.25 \mu m$ technology. Section VI presents the conclusions.

\section{REVIEW OF THE OPD METHODS}

Fig. 1 shows the flow of the OPD method in analog sizing. In each iteration, for each transistor of each candidate design, the input is the length and device biases. As shown by eqn (1), the $\mathrm{W}$ is computed by $\left\{L, I_{D S}, V_{d s}, V_{g s}, V_{b s}\right\}$.

$$
I_{D S}-I_{D S}\left(V_{G S}, V_{D S}, V_{B S}, \underline{W}, L\right)=0
$$


where $\mathrm{W}$ is the independent variable. Then, the estimated $\mathrm{W}$ for all the transistors are collected and the SPICE simulation using the L and the estimated $\mathrm{W}$ is done to obtain the performance of the candidate design parameters. The performances are sent to the optimization algorithm to start the next iteration.

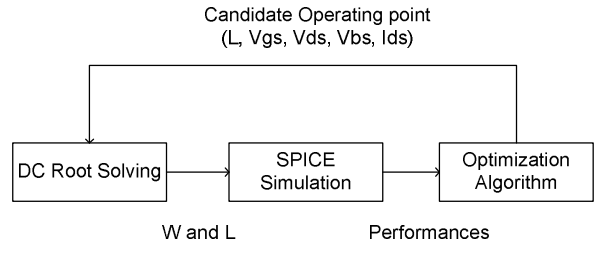

Fig.1. OPD-based analog sizing

It can be seen that the DC root solving is a critical problem in this flow. The methods to obtain W from eqn (1) can be classified into three main categories.

The first kind of method is to find $\mathrm{W}$ by directly solving eqn (1) $[3,4]$. First-order models can be computed easily, since an explicit equation for $\mathrm{W}$ exists. But it is too inaccurate for modern technologies. Hence, high-order models are necessary. Reference [3] suggests using the first-order solution as the starting point and then using SPICE-in-the-loop method to solve the equation. It also suggests scaling the variables to linearize strongly non-linear functions, which transforms eqn (1) to eqn (2).

$$
\log I_{D S}-\log I_{D S}\left(\log V_{g s}, \log V_{d s}, \log V_{b s}, \log \underline{W}, \log L\right)=0
$$

Because of using the SPICE simulation in the equation (no analytical form), using iteration methods to solve the equation are appropriate. In Section IV, we try Newton-Raphson method [8] and Golden Section Search method [9] to solve eqn (2) and found that a good $\mathrm{W}$ is difficult to be obtained (Section IV), especially in the 90nm technology (BSIM 4 model).

Another method to obtain $\mathrm{W}$ is regression. First, a set of training data are generated, and a regression model is constructed to predict $\mathrm{W}$ by the inputs $L, I_{D S}, V_{d s}, V_{g s}, V_{b s}$. ANN [7] is often considered as a powerful regressor. However, in our experiments, we found ANN is difficult to achieve high accuracy and generality at the same time (Section IV).

LUT is another kind of method to find W [5]. Many points are sampled in the $\left\{L, I_{D S}, V_{d s}, V_{g s}, V_{b s}\right\}$ space and stored in an LUT. There are different kinds of LUT-based methods, among which, the hierarchical look-up method is often considered as a very effective and efficient method [5]. For example, the method first finds the nearest left and right points of the given $V_{d s}$, and two candidate $V_{d s}$ are selected. In the LUT, there exist some points with these two $V_{d s}$. The algorithm again finds the nearest left and right points of the given $V_{g s}$ in the points with the two candidate $V_{d s}$. This process continues until the problem becomes a one-dimensional interpolation problem, e.g. the last variable is $I_{D S}$. At last, interpolation method is used to obtain the W. According to the experiments (Section IV), we found that the LUT method has relatively high accuracy when a large amount of samplings are used. But it costs a long look-up time and huge memory.

\section{THE OIOPD METHOD}

From the review of the above three methods using modern technologies, LUT is better than the other two methods. It can be seen that interpolation is an effective way. In the LUT method, there need 4 approximations before a one-dimensional interpolation. This process loses accuracy. To relieve this, LUT needs to include a large number of samples, which cause longer look-up time and more memory. On the other hand, for each operating point, the $\left\{L, I_{D S}, V_{d s}, V_{g s}, V_{b s}\right\}$ are determined. Therefore, selecting one variable to do the one-dimensional interpolation with $\mathrm{W}$ under the condition of the fixed other 4 variables (need on-line simulations) can considerably enhance the accuracy. The reason is that by the on-line simulation method, the other 4 variables are accurate, not by approximation. In OIOPD, we select $I_{D S}$ as the variable to do the interpolation with $\mathrm{W}$, using the determined $\left\{L, V_{d s}, V_{g s}, V_{b s}\right\}$. We first use different samples of $\mathrm{W}$ in the technology allowed range to generate the corresponding $I_{D S}$. Then, the estimated $\mathrm{W}$ can be interpolated using the generated W- $I_{D S}$ curve. Another advantage of on-line simulation is that the memory requirement problem is solved. The key problem is the necessary number of samples to achieve an accurate estimation using OIOPD and the comparison between the look-up time in the LUT method.

Fig. 2 shows two typical cases of the curve in a $0.25 \mu \mathrm{m}$ technology. It can be seen that the linearity between $\mathrm{W}$ and $I_{D S}$ is good in most part. Note that this 'linearity' is from a 'global' point of view. For a small segment of the curve, it may be highly nonlinear, but the whole curve can be represented by a linear regression function with small error. This observation means it may be not true that the more sampling points, the better accuracy. Because of the globally strong linearity, it is reasonable to use few samples. We compare the results of using 200 samples, 50 samples and 2 samples (the maximum and minimum width in a technology). The tested $\mathrm{W}, \mathrm{L}$ and biases are uniformly distributed in the allowed range of each technology. The $0.25 \mu \mathrm{m}$ and $0.18 \mu \mathrm{m}$ technologies use BSIM 3 model and the 90nm technology uses BSIM 4 model.
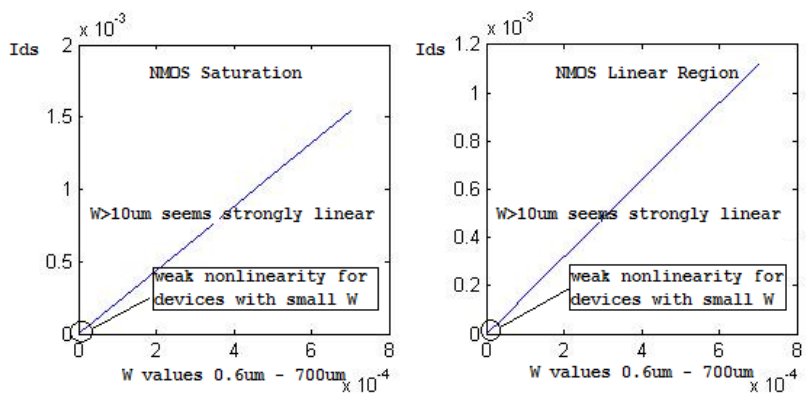

Fig. 2. Typical cases of W- $I_{D S}$ curve

According to the experiments, we found that the result of using 2 samples is comparable to, or even better than that of using 200 samples when the width is large than $10 \mu \mathrm{m}$. The reason is that the linearity is stronger in a global range compared with in a local range, as shown by Fig. 2. This is also an important reason to describe why directly solving eqn (2) to obtain W often cannot 
receive good results. For the transistors with width smaller than 10 $\mu \mathrm{m}$, using 2 samples have larger error. Using 50 samples or 200 samples all have good accuracy, and the result of using 200 samples is only a little better than using 50 samples, but it costs 4 times of the simulations. The following figure shows the errors of using 200 , 50 and 2 samples from $0.6 \mu \mathrm{m}$ to $50 \mu \mathrm{m}$ under typical device biases and $\mathrm{L}$ in a $0.25 \mu \mathrm{m}$ technology.

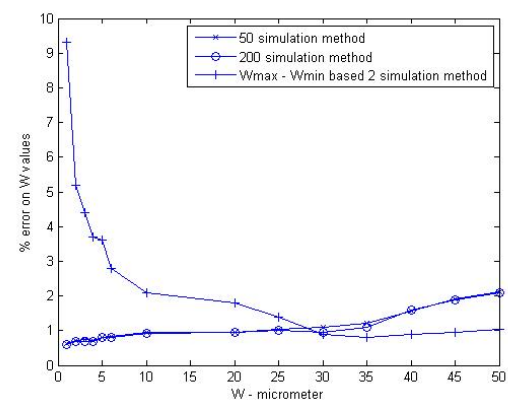

Fig. 3. Errors for different number of samples

Therefore, the OIOPD method uses the following rules to set the number of samples: (1) First uses the maximum and minimum $\mathrm{W}$ as the samples. (2) If the estimated transistor width is smaller than $10 \mu \mathrm{m}$, uses randomly distributed 50 samples within the minimum allowed $\mathrm{W}$ and $10 \mu \mathrm{m}$. On the other hand, it can be seen from Fig. 3 that even when the width is smaller than $10 \mu \mathrm{m}$, the error is within $10 \%$ in most cases (According to the experiments in Section IV, this is a small error). Hence, it is also reasonable to use 2 samples for all the transistors.

The flow diagram of OIOPD is shown in Fig. 4.

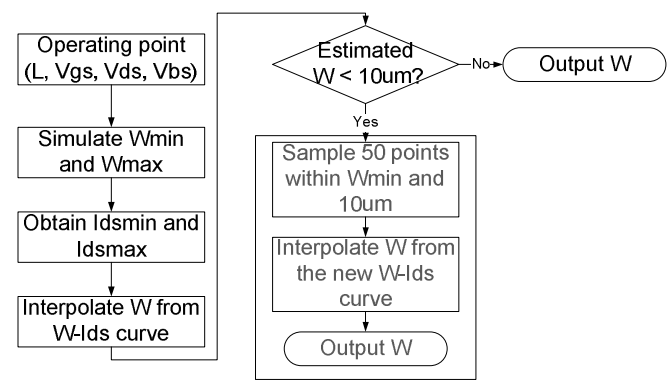

Fig. 4 Flow diagram of OIOPD

According to experiments, we found that the SPLINE interpolation [10] performs better than the linear and cubic interpolation. Hence, OIOPD uses the SPLINE interpolation.

\section{COMPARISONS WITH OTHER METHODS}

\section{A. Selection of the LUT}

It is known that there exists a trade-off between the accuracy, the look-up time and the memory requirement for different sizes of LUT. To make a fair comparison, a good LUT is necessary to be selected. The example below shows the selection of the LUT for a $0.18 \mu \mathrm{m}$ technology, and the selection of a $90 \mathrm{~nm}$ technology is done in the same way. We tried 3 LUT, and the accuracy, the lookup time for each transistor and the memory requirement of the 3 LUT are shown in Table I. For LUT, good indexing data structures can help to decrease the look-up time. In the experiments, we use the tree structure for the LUT. LUT 1 uses uniformly distributed 54 points within the range of $\mathrm{W}$ of the technology $(0.24 \mu \mathrm{m}$ to 800 $\mu \mathrm{m})$. LUT 2 uses 104 points of W, and LUT 3 uses 206 points of W. L has a range of $0.18 \mu \mathrm{m}$ to $10 \mu \mathrm{m}, V_{d s}, V_{g s}, V_{b s}$ have a range of 0 to $1.8 \mathrm{~V}$. LUT1 has 810,000 points, LUT2 has $1,560,000$ points and LUT3 has $3,090,000$ points. In the test process, 278 test points are selected, $40 \%$ of which are in the linear region, $60 \%$ of which are in the saturation region, and they are uniformly distributed. The experiments are run on a PC with Xeon processor and 8GB RAM memory in Linux system.

Table I. Different LUT for a $0.18 \mu \mathrm{m}$ technology

\begin{tabular}{|c|c|c|c|}
\hline Look-up Tables & Error & Look-up time & Memory \\
\hline LUT1 & $31 \%$ & $0.62 \mathrm{~s}$ & $55.6 \mathrm{MB}$ \\
\hline LUT2 & $23 \%$ & $1.19 \mathrm{~s}$ & $108.4 \mathrm{MB}$ \\
\hline LUT3 & $20 \%$ & $2.24 \mathrm{~s}$ & $205.4 \mathrm{MB}$ \\
\hline
\end{tabular}

It can be seen that LUT 2 makes the best trade-off. The look-up time for one transistor is not long to each LUT. But in the analog sizing, thousands times of this estimation are necessary, so the look-up time is an important factor. Hence, LUT 2 is chosen to compare with OIOPD.

\section{B. Comparisons of different methods}

Table II shows the comparisons of the Golden Section Search method, ANN, LUT and OIOPD. The tested W are also uniformly distributed in the allowed range of the technology. Traditional DC root solving methods include gradient-based ones and direct search ones. A typical method of gradient-based equation solving method is Newton-Raphson method [8]. In our experiments, for some DC points, the Newton-Raphson method causes SPICE un-convergence and cannot give a result, especially for the BSIM 4 model. Hence, Table II only includes the solution of the Golden Section Search method. We use the termination criterion in [9], and the absolute precision value is set to $10^{-8}$. ANN is selected in the same way as LUT. We compared tens of ANN with different training data, different number of neurons and different training methods. Table II shows the best of them, which uses 10 neurons in the hidden layer and the Levenberg-Marquardt algorithm [7].

From Table II, it can be seen that the results of ANN and Golden Section Search are far from anticipation. Through experiments, we found that for some points, the Golden Section Search method can receive comparable results with LUT, but more than $20 \%$ points even cannot converge. OIOPD has 10 times improvement on accuracy compared with LUT and 400 times improvement on efficiency. In addition, no extra memory is needed.

Table II. Comparisons of different method in a $0.18 \mu \mathrm{m}$ technology

\begin{tabular}{|c|c|c|c|}
\hline Methods & Error & Look-up time & Memory \\
\hline Golden Section & $872 \%$ & $4.39 \mathrm{~s}$ & $\mathrm{X}$ \\
\hline ANN & $143 \%$ & $0.30 \mathrm{~s}$ & $5 \mathrm{~KB}$ \\
\hline LUT & $23 \%$ & $1.19 \mathrm{~s}$ & $108.4 \mathrm{MB}$ \\
\hline OIOPD & $2.23 \%$ & $0.003 \mathrm{~s}$ & $\mathrm{X}$ \\
\hline
\end{tabular}

Comparison of LUT and OIOPD in a 90nm technology (BSIM 4 model) is shown in Table III. 
Table III. Comparison of LUT and OIOPD in a 90nm technology

\begin{tabular}{|c|c|c|c|}
\hline Methods & Error & Look-up time & Memory \\
\hline LUT & $23 \%$ & $2.32 \mathrm{~s}$ & $475.6 \mathrm{MB}$ \\
\hline OIOPD & $1.92 \%$ & $0.0021 \mathrm{~s}$ & $\mathrm{X}$ \\
\hline
\end{tabular}

From Table III, it can be seen that OIOPD has approximately 12 times improvement on accuracy and 1100 times improvement on efficiency. Hence, for nanometer technologies with more complex models, e.g. BSIM 4, the advantages of OIOPD are more obvious.

\section{AN ANALOG SIZING EXAMPLE USING OIOPD}

In this section, an example of using OIOPD to size complex analog circuit is provided. The gain-boosted folded-cascode amplifier in Fig. 5 is optimized by the OIOPD and LUT-based analog sizing method (the method to select the LUT is described in Section IV). We use a $0.25 \mu \mathrm{m}$ CMOS technology with $2.5 \mathrm{~V}$ power supply. The transistor lengths are allowed to vary between the minimum value allowed by the technological process, $0.25 \mu \mathrm{m}$, up to $10 \mu \mathrm{m}$. The transistor widths are changed between the minimum technology value, $0.6 \mu \mathrm{m}$, up to $700 \mu \mathrm{m}$. The capacitor values could change from $100 \mathrm{fF}$ to $20 \mathrm{pF}$. The design specifications are DC gain $>135 \mathrm{~dB}, \mathrm{GBW}>180 \mathrm{MHz}$, phase margin $>70^{\circ}$, gain $\operatorname{margin}<1$, output swing $>3.5 \mathrm{~V}$. All transistors should work in the saturation region. The optimization goal is the power consumption. The search algorithm is MSOEA [2]. Because of the influences of random numbers in evolutionary computation algorithms, 5 times are run for each method and the average results are shown in Table IV. The experiments are run on a PC with Xeon processor and 8GB RAM memory in Linux system.
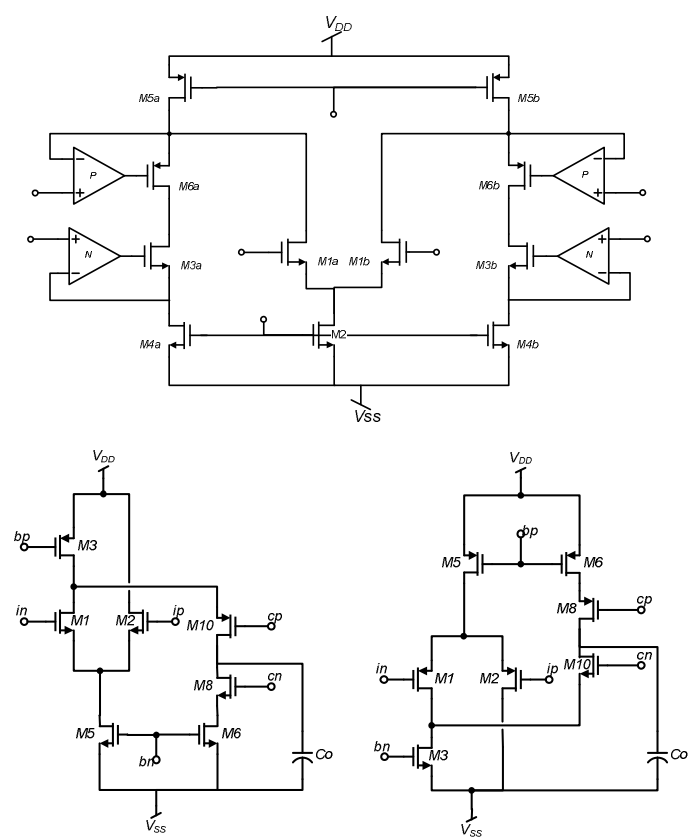

Fig. 5: (a) Gain-boosted folded-cascode amplifier, (b) P amplifier, and (c) N amplifier
Table IV. Results of the OIOPD and LUT method

\begin{tabular}{|c|c|c|c|}
\hline Method & $\begin{array}{c}\text { Objective } \\
\text { function }\end{array}$ & $\begin{array}{c}\text { Constraint } \\
\text { satisfaction }\end{array}$ & CPU time \\
\hline OIOPD & $6.51 \mathrm{~mW}$ & $23 / 23$ & $4654.4 \mathrm{~s}$ \\
\hline LUT & $9.20 \mathrm{~mW}$ & $21.4 / 23$ & $1.31 \mathrm{e}+6 \mathrm{~s}$ \\
\hline
\end{tabular}

From Table IV, it can be seen that the OIOPD method has better objective function values and satisfy all the 23 constraints in all the 5 runs. By the LUT method, only 2 runs satisfy all the constraints. This is because the OIOPD method can provide more accurate estimations of $\mathrm{W}$. The CPU time of OIOPD is also much smaller than that of using LUT. In order to get higher accuracy, the LUT need to include sufficient samples, and this increase the look up time, which is much longer than the time of simulating 2 extreme points. Because this example needs a large number of iterations, it can be seen that the time cost of the LUT method is impractical, but the OIOPD method also performs well (according to experiments, normally for a less complex circuit, the time cost of the LUT method is long but practical).

\section{CONCLUSIONS}

In this paper, an on-line interpolation operating-point driven (OIOPD) method is proposed. The key idea is to use very few online simulations and the interpolation method to estimate the width by the width-current curve with already determined length and voltage biases. OIOPD fully dominates the existing methods, which has 10 times improvement on accuracy, 300-1100 times improvement on efficiency and no extra memory is required. In addition, unlike the LUT method and the ANN method, almost no parameters are necessary to be tuned. Tests on single transistor and complex circuit and comparisons show that the OIOPD method awakes the operating-point driven-based analog sizing in modern technologies.

\section{REFERENCES}

[1] D. Mueller et al., 2007. "Trade-off Design of Analog Circuits Using Goal Attainment and "Wave Front" Sequential Quadratic Programming", Proc. Of DATE, pp. 16-20.

[2] B. Liu, et al., 2009. "A Memetic Approach to the Automatic Design of High Performance Analog Integrated Circuits", ACM TODAES, 14(3), Article 42.

[3] F. Leyn et al., 1998. "An Efficient DC Root Solving Algorithm with Guaranteed Convergence for Analog Integrated CMOS Circuits", Proc. of ICCAD, pp. 304-307.

[4] F. Leyn et al., 1997. "Analog Circuit Sizing with Constraint Programming Modeling and Minimax Optimization", Proc. of ISCAS, pp. 1500-1503.

[5] T. McConaghy et al., 2009. "Trustworthy, variation-aware structural synthesis of analog circuits via structural homotopy", IEEE TCAD, pp. 1281-1294.

[6] H. Graeb, 2007. “Analog Design Centering and Sizing”, Spring.

[7] PD Wasserman, 1988. Neural computing: theory and practice. New York: Van Nostrand Reinhold.

[8] C. Kelley, 2003. "Solving Nonlinear Equations Using Newton's method", SIAM.

[9] W. Press et al., 1999. "Numerical Recipes in C, The Art of Scientific Computing ( $2^{\text {nd }}$ edition)", Cambridge University Press.

[10] C. Boor, 1978. "A Practical Guides to Splines", Springer-Verlag. 